\title{
Reproducibility of the heat/capsaicin skin sensitization model in healthy volunteers
}

This article was published in the following Dove Press journal:

Journal of Pain Research

6 November 2013

Number of times this article has been viewed

\author{
Laura F Cavallone' \\ Karen Frey' \\ Michael C Montana' \\ Jeremy Joyal' \\ Karen J Regina' \\ Karin L Petersen ${ }^{2}$ \\ Robert W Gereau IV' \\ 'Department of Anesthesiology, \\ Washington University in St Louis, \\ School of Medicine, St Louis, MO, \\ USA; ${ }^{2}$ California Pacific Medical \\ Center Research Institute, San \\ Francisco, CA, USA
}

Video abstract

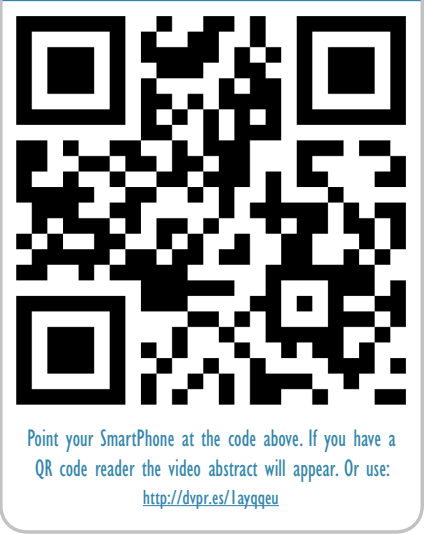

Correspondence: Laura F Cavallone Campus Box 8054, Department of Anesthesiology, Washington University in St Louis, 660 S Euclid Ave, St Louis, MO 63II0, USA

Tel +l $3 \mid 43622358$

Fax + I 314362 |185

Email cavallol@wustl.edu
Introduction: Heat/capsaicin skin sensitization is a well-characterized human experimental model to induce hyperalgesia and allodynia. Using this model, gabapentin, among other drugs, was shown to significantly reduce cutaneous hyperalgesia compared to placebo. Since the larger thermal probes used in the original studies to produce heat sensitization are now commercially unavailable, we decided to assess whether previous findings could be replicated with a currently available smaller probe (heated area $9 \mathrm{~cm}^{2}$ versus $12.5-15.7 \mathrm{~cm}^{2}$ ).

Study design and methods: After Institutional Review Board approval, 15 adult healthy volunteers participated in two study sessions, scheduled 1 week apart (Part A). In both sessions, subjects were exposed to the heat/capsaicin cutaneous sensitization model. Areas of hypersensitivity to brush stroke and von Frey (VF) filament stimulation were measured at baseline and after rekindling of skin sensitization. Another group of 15 volunteers was exposed to an identical schedule and set of sensitization procedures, but, in each session, received either gabapentin or placebo (Part B).

Results: Unlike previous reports, a similar reduction of areas of hyperalgesia was observed in all groups/sessions. Fading of areas of hyperalgesia over time was observed in Part A. In Part B, there was no difference in area reduction after gabapentin compared to placebo.

Conclusion: When using smaller thermal probes than originally proposed, modifications of other parameters of sensitization and/or rekindling process may be needed to allow the heat/ capsaicin sensitization protocol to be used as initially intended. Standardization and validation of experimental pain models is critical to the advancement of translational pain research.

Keywords: experimental pain model, hyperalgesia, peripheral sensitization, central sensitization

\section{Introduction}

A reliable method to produce hyperalgesia and allodynia in human healthy volunteers could have a crucial role in the development of novel analgesic compounds by helping to bridge the gap between animal pain models and chronic pain patients. ${ }^{1,2}$ Hyperalgesia and allodynia are common symptoms experienced in chronic pain syndromes ${ }^{3,4}$ and are thought to depend on central nervous system sensitization, which is maintained both by central mechanisms and ongoing peripheral stimuli. ${ }^{3-8}$ However, creating the experimental conditions to reliably induce central sensitization and test the reversal of established hyperalgesia is a complex task. ${ }^{9}$ The experimental model must be safe, tolerable, and induce sensitization lasting long enough to allow testing of both prevention of hyperalgesia and reversal of established hypersensitivity without creating permanent damage. 
One well characterized human experimental model of cutaneous sensitization is the heat/capsaicin model. ${ }^{1,10}$ This method uses the initial application of heat with a Peltier-type thermal probe, then topical capsaicin cream, and subsequent periodic thermal rekindling procedures to induce stable, reproducible, and long-lasting (up to 4 hours) hyperalgesia and secondary hypersensitivity in healthy human volunteers and patients. ${ }^{1,2,10-16}$

One advantage of the heat/capsaicin model is the ability to generate hypersensitivity of sufficient duration to test the onset and magnitude of drug analgesia without creating unacceptable discomfort, tissue injury, or long-lasting effects. ${ }^{110,17}$ The heat/capsaicin sensitization model has been applied successfully to demonstrate the ability of gabapentin, among other drugs, to reduce cutaneous hyperalgesia. These encouraging results, obtained with gabapentin, which is clinically effective in treating neuropathic pain, proved that the model could be used to predict clinical antihyperalgesic efficacy. ${ }^{16,18,19}$ However, the reproducibility of all experimental pain models is influenced by multiple factors that may affect within-day and between-day variability of the subjects' responses, ${ }^{17,20,21}$ and practical and logistical issues may limit the consistency of results between observers and/ or laboratories.

The size of the area directly stimulated with heat and capsaicin (area of "primary hyperalgesia"), and the resulting size of the surrounding area of secondary hyperalgesia may be critical factors in the reproducibility and applicability of this model. ${ }^{20,22}$ When first reported, the heat/capsaicin sensitization model used thermal stimulators with a heated surface area of $12.5-15.7 \mathrm{~cm}^{2} .1,2,10-12,15,16$ The heat/capsaicin model, as originally proposed, has become widely popular and extensively utilized in research; the methods have been reported in publications, lectures, and refresher courses at international meetings. ${ }^{1,10,23,24}$ However, since publication of the original report, the thermal probes that were utilized are no longer commercially available. The largest Peltier probe that is currently available has a heated surface area of $9 \mathrm{~cm}^{2}$.

As the intensity of the stimulus used to induce the cutaneous hyperalgesia has been shown to affect both extent and duration of the hyperalgesia, ${ }^{25,26}$ using the smaller thermode $\left(9 \mathrm{~cm}^{2}\right)$ may affect the size and duration of the area of secondary hyperalgesia both by heating a smaller area of skin and by providing a smaller contact surface to capsaicin cream (in this model the area covered with capsaicin cream corresponds to the thermode outline). This prompted us to question the possibility to reliably reproduce the model with a smaller area of peripheral sensitization. We knew that some researchers had been able to reproduce heat/capsaicin sensitization with smaller Peltier probes by utilizing modified procedures. The original papers by Petersen and Dirks ${ }^{1,10}$ were quoted as a source of the model in multiple studies by different groups in which, however, the protocol was significantly modified from the original, with different exposure time to capsaicin and/or different procedures of application or maintenance of the stimulus. ${ }^{27-30}$

Prior studies of analgesic responsiveness should be replicated before adopting the $9 \mathrm{~cm}^{2}$ thermal probe as the standard for inducing heat/capsaicin cutaneous sensitization with this model, and, if a different heat/capsaicin model needs to be proposed as a standard, this would need to be validated on the basis of rigorous observations. The sequential application of heat and capsaicin will not always or necessarily produce reliable and stable sensitization. It is not known what are the essential elements of a model that associates heat and capsaicin to consistently create long lasting cutaneous sensitization; these factors may vary interindividually and also depend on the stimulation site and the outcomes observed. Using the $9 \mathrm{~cm}^{2}$ Peltier thermode to induce cutaneous sensitization, the present study investigated the reproducibility of the heat/ capsaicin model as originally proposed $\mathrm{d}^{1,10,16}$ by assessing: a) the size and duration of secondary hyperalgesia; and b) the antihyperalgesic response to a single oral dose of $1,200 \mathrm{mg}$ gabapentin (the same single dose of gabapentin that had significantly decreased areas of hyperalgesia produced with the original heat/capsaicin model compared to placebo). ${ }^{16}$

\section{Materials and methods Study subjects and design}

The study was conducted in accordance with ethical principles of Good Clinical Practice and the Declaration of Helsinki and its guidelines, and approved by Washington University in St Louis Institutional Review Board.

Subjects provided written informed consent after the study procedures were explained and their questions were answered, and before study procedures were initiated. A list of the inclusion and exclusion criteria, which were identical to the criteria utilized in the original study that we were seeking to replicate, ${ }^{16}$ is provided in Table 1 , and demographic data are shown in Table 2.

In a screening/training session preceding the first study session, subjects were familiarized with the study procedures, including the induction of sensitization with sequential application of heat and capsaicin (Figure 1A). During the first study session, heat/capsaicin sensitization was induced and 
Table I Inclusion and exclusion criteria

Inclusion criteria
I. Male or nonpregnant, non-nursing female
2. I8-50 years old
3. Good general health with no remarkable medical conditions
(eg, liver, kidney, heart, or lung failure)
4. Body mass index between $20-33$
5. Unmedicated (excludes contraceptives)
6. Willing to comply with study guidelines as outlined in protocol
7. Willing to provide informed consent
Exclusion criteria
1. Anatomical malformation of upper extremities
2. Status post recent trauma or chronic lesions on either forearm
3. Medication use (excludes contraceptives)
4. History of allergy or intolerance to capsaicin
5. History of allergy or intolerance to gabapentin
6. History of addiction to drugs or alcohol (prior or present addiction
or treatment for addiction)
7. History of chronic pain syndromes
8. Pregnant and nursing females
9. Heat Pain Detection Threshold greater than $47^{\circ} \mathrm{C}$ at baseline
10. Unable to achieve cutaneous sensitization with heat/capsaicin during
the training session

maintained by rekindling the stimulation site with heat four times at regular intervals over the 4 hour study day. Areas of secondary allodynia, and hyperalgesia to foam brush and VF filament (primary outcomes); heat pain detection threshold (HPDT) in the stimulation site (secondary outcomes); and the painfulness of thermal stimulation (PTS) on nonsensitized skin (secondary outcomes) were determined multiple times during the study day. In all the study sessions, measurements were performed before the induction of sensitization (M0 in Figure 1B), after the first rekindling (RK1) (M1 in Figure 1B) and after the fourth rekindling (RK4) (M2 in Figure 1B). The study consisted of two parts:

Part A evaluated the natural history of sensory changes following heat/capsaicin sensitization. Subjects in this group underwent the procedures and measurements described above during two study sessions separated by at least 1 week to allow the skin to recover between sessions. In this group

Table 2 Study subjects' demographics

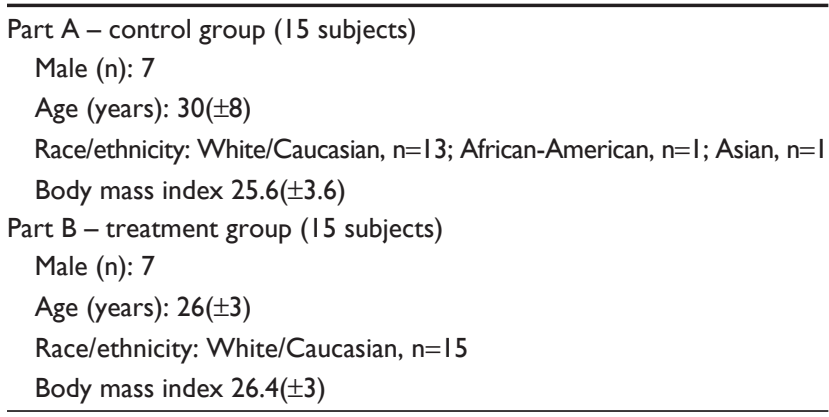

only, additional area, HPDT, and PTS measurements were repeated after RK2 and after RK3 to better follow the sensory changes. Subjects in Part A will be referred to as the "control group".

Part B was a double-blind, randomized, crossover study in healthy volunteers to evaluate the response to gabapentin using the heat/capsaicin model. On the first study session, subjects were randomly assigned to receive a single oral dose of gabapentin $(1,200 \mathrm{mg})$ or placebo immediately after induction of the heat/capsaicin sensitization as previously described by Dirks et al. ${ }^{16}$

Outcome measures were collected as described above (baseline, pre-drug and post-drug; for detail see Figure 1B). During the second study session, subjects underwent the same procedures but received the treatment that they had not received during their first session. Sessions were 1 week apart to allow washout of the treatment administered in the first session. Areas obtained after gabapentin/placebo administration (M2 in Figure 1B) were compared to postsensitization baseline areas (M1 in Figure 1B) and the results obtained with gabapentin and placebo were compared. Subjects in Part B will be referred to as the "gabapentin/placebo group".

Sample size estimate was based on the previously published between-days coefficients of variation of the areas of hyperalgesia obtained with this model. ${ }^{1}$ In the methodological study aimed at validating the heat/capsaicin model, ${ }^{1}$ the authors calculated sample sizes needed to detect treatment differences with this model, based on the coefficients of variation of areas of hyperalgesia within-day and between-day. Assuming for our study the same coefficients of variation, and planning a between-days comparison and a cross-over design, we recalculated the sample size to obtain a probability of $80 \%$ that the study could detect a treatment difference at a two-sided 0.05 significance level, if the true difference in area reduction between treatments (gabapentin and placebo) was at least $30 \%$ (in the original study, comparing gabapentin and placebo with this model, the observed difference in area reduction was about $70 \%$ ). Our sample size calculation indicated that 15 subjects were necessary in the treatment group. An equal number of subjects were enrolled in the control group for comparison.

Forty-nine subjects provided written consent and were enrolled in the study. Seventeen subjects did not meet inclusion criteria during the screening session and two more were excluded during the study as they met exclusion criteria after enrollment. The remaining 30 subjects completed Part A $(n=15)$ or Part B $(n=15)$ of the study. The 15 subjects who took part in Part B of the study were assigned 


\section{A) Timeline of screening/training day procedures}

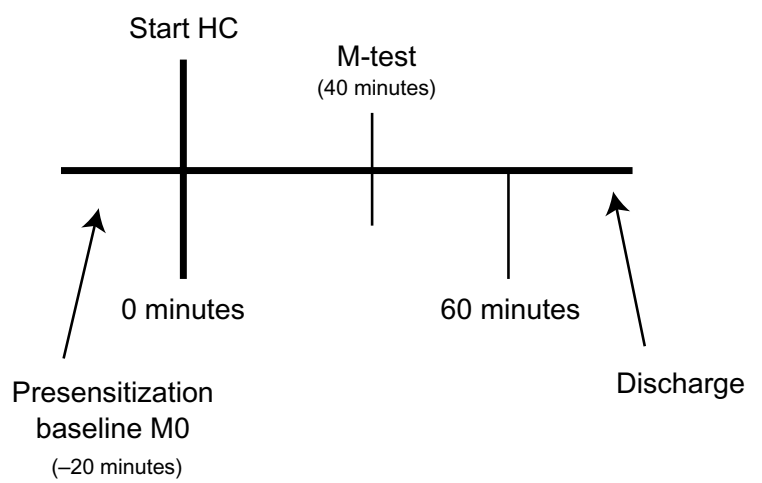

\section{B) Timeline of study day procedures - sessions 1 and 2}

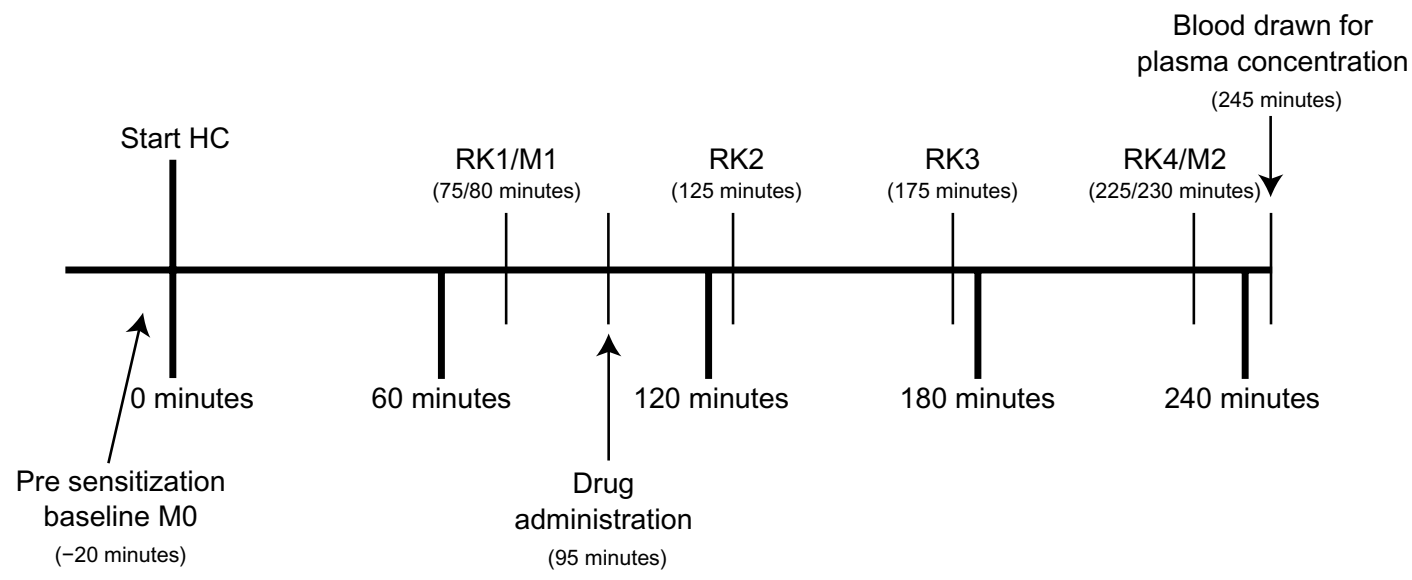

Figure I Timeline of training and study sessions. The procedures performed in each session are illustrated. (A) screening/training day and (B) study days.

Notes: In the control group there was no drug administration, and additional sets of the same measurements described below (M) were taken after RK2 and RK3. In the gabapentin/placebo group, on session I and 2, subjects were randomly assigned to receive gabapentin or placebo. M refers to a set of measurements (mapping of area of hyperalgesia/allodynia, heat pain detection threshold, pain during thermal stimulation).

Abbreviations: $\mathrm{HC}$, heat/capsaicin sensitization; RK, rekindling.

to receive placebo or gabapentin on their first study session by a computer generated randomization table. Subjects and investigators directly involved in data collection and analysis were blinded to the treatment allocation. The randomization sequence was provided by the investigational pharmacy, and a research nurse not directly participating in the study managed the schedule and assigned subjects to interventions. Of the 15 subjects who completed Part B, one subject was excluded from the analysis because the blood sample collected on the placebo day contained gabapentin, indicating a possible confusion in the blood sample analysis or erroneous administration of gabapentin on the placebo day. A flow diagram of enrollment and allocation of subjects in the study is shown in Figure 2.

\section{Study measurements and procedures}

All study procedures were maintained identical to the original protocol $^{16}$ and performed by the same investigator (KF) in a quiet environment, at controlled room temperature, with subjects comfortably sitting in a hospital bed or recliner with armrests.

\section{Heat/capsaicin sensitization procedure}

Thermal stimulations were applied in a precise and controlled manner using a Medoc Advanced Thermal Stimulator (Medoc Ltd., Ramat Yishai, Israel) driving a $9 \mathrm{~cm}^{2}$ thermode. An area of the skin on the volar surface of the dominant forearm of each subject was chosen, and the distance of the center of this area from the elbow crease and from the wrist 


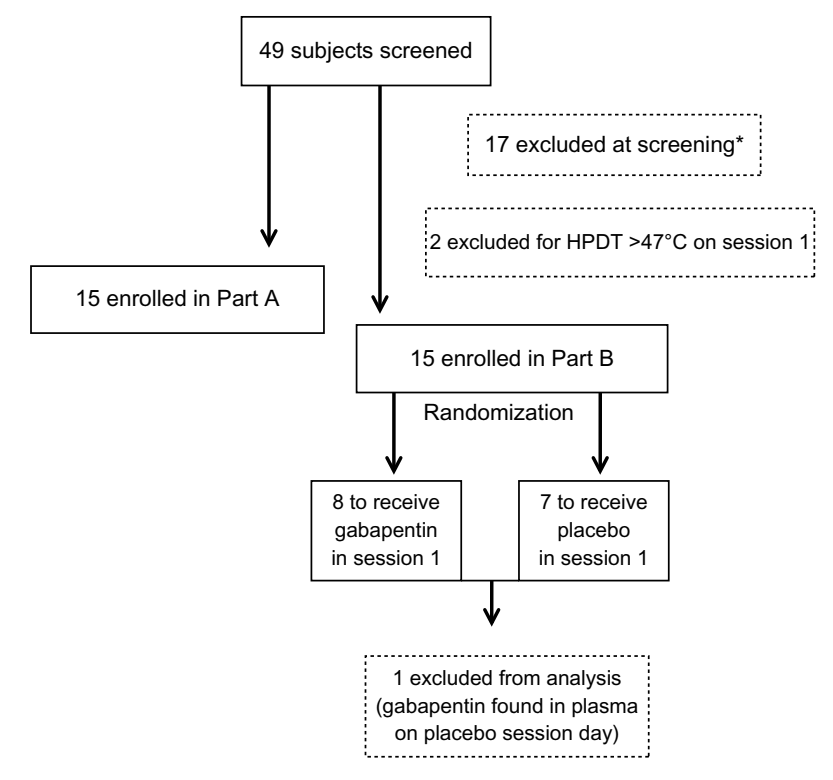

Figure 2 Enrollment and allocation of participants in the study.

Note: *Reasons for exclusions on screening day: $5=$ HPDT $>47^{\circ} \mathrm{C}$; $9=$ unable to achieve sensitization (area); I= admitted history of drug abuse; I= showed lesions on forearm at physical examination; I= body mass index $>33$.

Abbreviation: HPDT, heat pain detection threshold.

crease was recorded to keep the site of the area consistent between sessions. Heat was applied to this previously delimited area using the thermode, which ramped from $32^{\circ} \mathrm{C}$ to $45^{\circ} \mathrm{C}$ at a rate of $1^{\circ} \mathrm{C}$ per second and then held at $45^{\circ} \mathrm{C}$ for 5 minutes. During these 5 minutes, subjects used an electronic handheld device to rate their pain every minute by moving a bar on a $0-10$ visual analog scale (VAS), where 0 indicates "no pain" and 10 indicates "the most intense pain imaginable." The handheld device was connected to a computer that recorded the ratings on a $0-100$ scale. The heating procedure was followed by immediate application of capsaicin cream (Capzasin-HP cream [previously 0.075\%, now marketed as 0.1\%], Chattem Inc., Chattenooga, TN, USA) covering the heated surface (outline of thermode). The cream was left on for 30 minutes and then gently wiped off in one single motion, taking care in avoiding rubbing the cream into the skin. Approximately $0.5 \mathrm{oz}$ of cream was required to cover the entire surface completely. Subjects were asked to rate their pain on the handheld VAS at the start of the 30 minute period and then for every 5 minutes until the cream was removed.

\section{Rekindling}

On study days, hypersensitivity was maintained by "rekindling" the site of heat/capsaicin application. This was accomplished by restimulating the previously treated skin four times at 45-minute intervals with the thermode increasing from $32^{\circ} \mathrm{C}$ to $40^{\circ} \mathrm{C}$ at a rate of $1^{\circ}$ per second and held at $40^{\circ} \mathrm{C}$ for 5 minutes. Subjects rated their pain on a continuous VAS during rekindling.

\section{Mapping of areas of hypersensitivity}

The borders of secondary allodynia and hyperalgesia were mapped using a 1 inch foam brush and a $26 \mathrm{~g} \mathrm{VF}$ filament (26 g bending force [North Coast Medical, Gilroy, CA, USA]). Subjects were asked to close their eyes while the brush was applied along four linear paths between the thermode outline and: 1) the antecubital fossa; 2) the wrist; 3) the lateral forearm; and 4) the medial forearm. Stimulation was started distant from the borders of the heated area and worked closer in $5 \mathrm{~mm}$ steps at 1 -second intervals. Subjects were instructed to recognize a "distinct change in sensation" such as "increased burning, tenderness, more intense pricking, or an unpleasant sensation," and that location was marked. This procedure was then repeated with the VF filament. Areas of hypersensitivity were calculated (in $\mathrm{cm}^{2}$ ) as the distance between the farthest points marked on the rostral/ caudal axis multiplied by the distance between the farthest points marked on the medial/lateral axis. The presence of cutaneous hypersensitivity was assessed before the induction of sensitization (M0 in Figure 1A and B), after RK1 (M1 in Figure 1B), and after RK4 (M2 in Figure 1B). Two additional measurements (after RK2 and after RK3) were taken in Part A of the study.

\section{Heat pain detection threshold}

The threshold for heat pain detection was determined by using a thermal ramp protocol on the marked location on the volar surface of the forearm. The temperature applied through the thermode was increased from $32^{\circ} \mathrm{C}$ to the $50^{\circ} \mathrm{C}$ safety cutoff at $1{ }^{\circ} \mathrm{C}$ per second. Subjects were asked to turn off the heated thermode by depressing a button at "the lowest temperature that they perceived as painful." Four thermal ramps were performed 10 seconds apart and their median value was calculated. To avoid testing individuals whose pain threshold approached the safety cutoff, subjects with HPDTs greater than $47^{\circ} \mathrm{C}$ were excluded from the study. HPDT was assessed before the induction of sensitization (M0 in Figure 1A and B), after RK1 (M1 in Figure 1B), and after RK4 (M2 in Figure 1B). Two additional measurements (after RK2 and after RK3) were taken in Part A of the study.

\section{Pain during thermal stimulation}

Acute pain was induced by a 1 -minute $45^{\circ} \mathrm{C}$ heat stimulus on a marked location on the upper nondominant arm (deltoid). Subjects were asked to rate their pain intensity during the 
1-minute heat stimulus continuously using the electronic VAS, as described above. PTS was assessed before the induction of sensitization (M0 in Figure 1A and B), after RK1 (M1 in Figure 1B), and after RK4 (M2 in Figure 1B). In Part A of the study, PTS was also assessed after RK2 and after RK3.

\section{Measurement of plasma concentration of gabapentin}

All subjects in the drug/placebo group had a blood sample $(5 \mathrm{~mL})$ collected from the nondominant arm to assess gabapentin plasma concentration at 150 minutes after drug/ placebo administration (for preservation of blinding in the protocol, the sample was collected in both sessions). Plasma was frozen for later analysis.

Gabapentin was quantitated using LC/MS/MS (liquid chromatography and tandem mass spectrometry). Plasma samples were thawed, vortexed, and aliquots of $475 \mu \mathrm{L}$ of sample, standard or quality control sample, were transferred into a deep well 96-well plate. To each well were added $0.25 \mathrm{~mL}$ of $1 \mathrm{M}$ sodium phosphate buffer and $25 \mu \mathrm{L}$ of a $0.5 \mu \mathrm{g} / \mathrm{mL}$ Gabapentin D10 internal standard solution prepared in water (Sigma-Aldrich, St Louis, MO, USA). Samples, standards, and quality controls were then applied to a Bond Elut C18 solid phase extraction plate (Agilent Technologies, Böblingen, Germany), which had been prepared by washing first with $1.0 \mathrm{~mL}$ of methanol and then with $1.0 \mathrm{~mL}$ of $1 \mathrm{M}$ sodium phosphate buffer. After applying the samples to the extraction plate it was washed with $1.0 \mathrm{~mL}$ of $0.1 \mathrm{M}$ sodium phosphate buffer and then with $1.0 \mathrm{~mL}$ of $0.1 \mathrm{M}$ hydrochloric acid. Samples were eluted with $1.0 \mathrm{~mL}$ of methanol and the plate dried down at $30^{\circ} \mathrm{C}$ under a gentle flow of nitrogen. Samples were reconstituted with $500 \mu \mathrm{L}$ of mobile phase: $95 \% 4.5 \mathrm{mM}$ ammonium acetate in water $/ 5 \%$ $4.5 \mathrm{mM}$ ammonium acetate in acetonitrile. The deep well plate was shaken for 10 minutes and $200 \mu \mathrm{L}$ was transferred to a 96-well autosampler plate. Calibrators ( 8 concentrations over the range 0.5 to $50 \mathrm{ng} / \mathrm{mL}$ ) and three quality control samples $(3,15$, and $30 \mathrm{ng} / \mathrm{mL})$ in human plasma were prepared along with experimental samples.

Instrumental analysis was performed on an API 4000 QTRAP triple-quadropole mass spectrometer (Life Technologies, Carlsbad, CA, USA) equipped with a Turbo IonSpray Source. A Shimadzu UFLC system (Shimadzu Corporation, Kyoto, Japan) that consisted of a CMB-20A system controller, two LC-20ADXR pumps, a DGU-20A3 degasser, a SIL-20AC autosampler, and a CTO-20A column oven was used to deliver the samples to the detector. An external two-way Valco valve (Valco Instruments Co. Inc., Houston, TX, USA) was utilized to direct high-performance liquid chromatography flow to waste before and after column elution of analytes of interest. Chromatographic separation was performed on a Kinetix $2.6 \mu \mathrm{m}$ C18 analytical (2.1 by $100 \mathrm{~mm}$ ) column (Phenomenex Inc., Torrance, CA, USA) with a Security Guard ULTRA C18 guard cartridge for $2.1 \mathrm{~mm}$ ID columns installed prior to the analytical column (Phenomenex). Before each injection, the needle was washed with $10 \%$ isopropanol. Mobile phase A was $4.5 \mathrm{mM}$ ammonium acetate in water and mobile phase $\mathrm{B}$ was $4.5 \mathrm{mM}$ ammonium acetate in acetonitrile. Mobile phase was delivered at initial conditions of $5 \% \mathrm{~B}$ at a flow rate of $0.3 \mathrm{~mL} /$ minute with the following time program: hold at $5 \% \mathrm{~B}$ for 0.6 minutes followed by a linear gradient over 0.65 minutes between $5 \%$ and $95 \%$ B; hold at $95 \%$ B for 1.25 minutes; mobile phase composition is then brought back down to initial condition of $5 \%$ over 0.01 minutes. The column was re-equilibrated with 5\% B from 2.51 to 4.0 minutes. The retention time for gabapentin was 2.2 minutes under these conditions. Both Q1 and Q3 quadrupoles of the mass spectrometer were optimized to unit mass resolution. The instrument was operated in positive-ion mode with an ion spray voltage of 5,500 volts and a temperature of $450^{\circ} \mathrm{C}$. The curtain gas was set at 20 pound per square inch (psi), ion source gas 1 at 30 psi, gas 2 at 40 psi, and the collision gas was set to medium. Gabapentin and the internal standard were detected by multiple reaction monitoring, using the transitions $\mathrm{m} / \mathrm{z} \quad 172 \rightarrow 137$ and $182 \rightarrow 104$, respectively. Quantitation was performed in Analyst software version 1.5.2 (Life Technologies).

\section{Statistical analysis}

Our primary outcome measure was the size of the areas of hyperalgesia to punctate stimuli (VF filament stimulation) and dynamic tactile allodynia (brush stimulation) outside the area directly exposed to heat and capsaicin, ie, the areas of secondary hyperalgesia. Secondary outcome measures included HPDT in the area directly stimulated with heat and capsaicin (ie, the area of primary hyperalgesia), painfulness of the rekindling procedures quantified by visual analog scale (handheld device on a $0-10$ scale translated in electronic VAS on a $0-100$ scale), and painfulness of a one minute $45^{\circ} \mathrm{C}$ stimulation in normal skin (pain during thermal stimulation, PTS as quantified by VAS).

Data were analyzed with Analyse-it (Analyse-it Software, Ltd., Leeds, UK) version 2.26. Appropriate data 
transformations and nonparametric methods were used as indicated. Measurements of areas of hypersensitivity were normalized for each subject using the baseline measurements, so that the final outcome is expressed as the percent of baseline area. Measures of area changes, HPDT, and VAS during thermal stimulation (PTS) are expressed as medians as well as 1 st and 3rd quartiles of the values for each subject at each chosen time point. Measures of VAS during rekindling procedures are expressed as mean and standard deviation. Comparisons of data sets were performed with Student's $t$-test and Wilcoxon test for paired data, and one-way ANOVA and Friedman test for repeated measures. A $P$ less than 0.05 was considered statistically significant. For all analyses, careful attention was given to whether the data satisfied the distributional and model-specific assumptions of the procedures used.

\section{Results}

\section{Areas of secondary hypersensitivity to VF and foam brush stroke stimulation}

\section{Study sessions (Parts A and B)}

Areas of hypersensitivity to VF stimulation and brush stroke and red flare as expected were obtained after sensitization with heat and capsaicin in all subjects in both Parts A and B of this study. However, in contrast to what has been previously reported, ${ }^{1,10,16}$ it was not possible to maintain these areas as stable with rekindling procedures throughout the study day. The areas of hypersensitivity tended to fade spontaneously in the absence of pharmacological intervention (Part A), and no significant difference was observed in reduction of areas of secondary hyperalgesia and allodynia after treatment with gabapentin compared to placebo (Part B).

After gabapentin administration, post-RK4 (M2 in Figure 1B), the areas of secondary hyperalgesia to VF stimulation and allodynia to brush stroke were reduced to $65 \%$ $(34.3 \%-85.3 \%)$ and $32.8 \%(0 \%-76.7 \%)$, respectively, of the areas measured at post-RK1 (M1 in Figure 1B). After placebo administration, areas mapped by VF stimulation and brush stroke were reduced to $78.4 \%(46.4 \%-84.9 \%)$ and $42.9 \%$ (34.6\%-56.2\%), respectively. Similar results were observed in the control group (Part A) that did not receive either placebo or drug (in Session 1, post-RK4 VF areas were reduced to $56 \%$ [34.6\%-87\%] of post-RK1 area, and brush areas were reduced to $40.3 \%$ [31.6\%-79.6\%] of post-RK1 area. In Session 2, VF areas were reduced to $67.1 \%$ [41.5\%-86.2\%], and brush areas were reduced to $55 \%$ [22.5\%-73.3\%\%]). These reductions of the areas of hypersensitivity were not significantly different between treatment sessions in any group (Figure $3 \mathrm{~A}-\mathrm{D})$.

\section{Heat pain detection thresholds}

HPDTs were measured to evaluate increased heat sensitivity in the area of primary sensitization. HPDTs at M0 (Figure 1B) were not different between sessions in any group.

\section{Training session}

On the training day, subjects enrolled both in Part A and Part B of the study showed significantly decreased HPDTs immediately after induction of sensitization with heat and capsaicin (M-test in Figure 1A) compared to baseline presensitization $(P<0.0001$ and $P<0.0004$ for subjects in Part A and Part $\mathrm{B}$, respectively).

\section{Study sessions}

Part A

In the control group, baseline HPDTs before sensitization (M0 in Figure 1B) were not different between treatment sessions $(P=0.8)$. HPDTs postsensitization were not different from $\mathrm{M} 0$ at any time point (post-RK1 through RK4), or in any of the two study sessions (Session $1 P=0.16$ and Session $2 P=0.09$ ).

\section{Part B}

In the gabapentin/placebo group, baseline HPDTs before sensitization (M0 in Figure 1B) were also not different between treatment sessions $(P=0.58)$.

After RK1 (M1 in Figure 1B), HPDTs were significantly lower than $\mathrm{M} 0$ in the placebo session $(P=0.03)$, but were not significantly different to $\mathrm{M} 0$ in the gabapentin session $(P=0.28)$. On the placebo day, post-drug HPDTs (M2 in Figure 1B) showed no difference with M1 $(P=0.9)$ and remained significantly lower than $\mathrm{M} 0(P=0.002)$ (Figure 4$)$.

In the gabapentin session, post-drug HPDT measurements at M2 were not different compared to both M0 and to M1 ( $P=0.08$ and $P=0.7$, respectively).

Post-drug (M2) HPDTs were not significantly different in the gabapentin session compared to the placebo session $\left(41.7^{\circ} \mathrm{C}\right.$ $\left[40.8^{\circ} \mathrm{C}-42.8^{\circ} \mathrm{C}\right]$ versus $\left.41.4^{\circ} \mathrm{C}\left[40.6^{\circ} \mathrm{C}-42.4^{\circ} \mathrm{C}\right] ; P=0.41\right)$.

\section{VAS scores during rekindling procedures}

Study session (Part A and Part B)

In both Part A and Part B of the study, pain reported by subjects during RK1 and RK4, respectively, remained constant throughout the 5 minute procedure in both sessions (analysis 


\section{No treatment (part A - control group)}

Area of secondary hyperalgesia

(to VF stimulation)

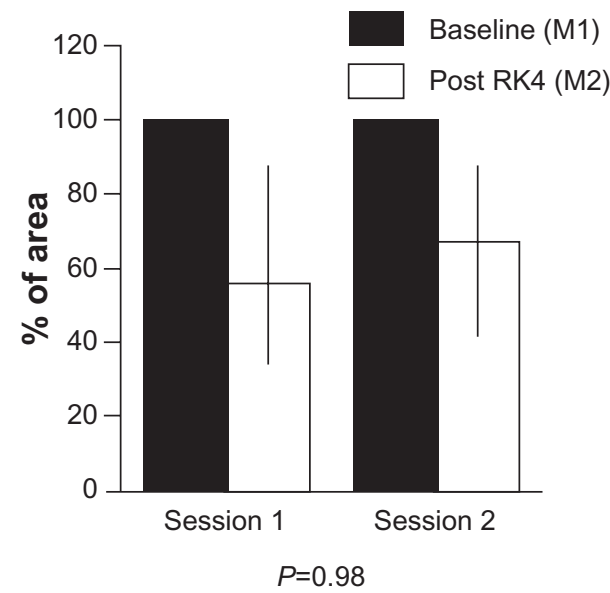

Area of secondary allodynia

(to brush stimulation)

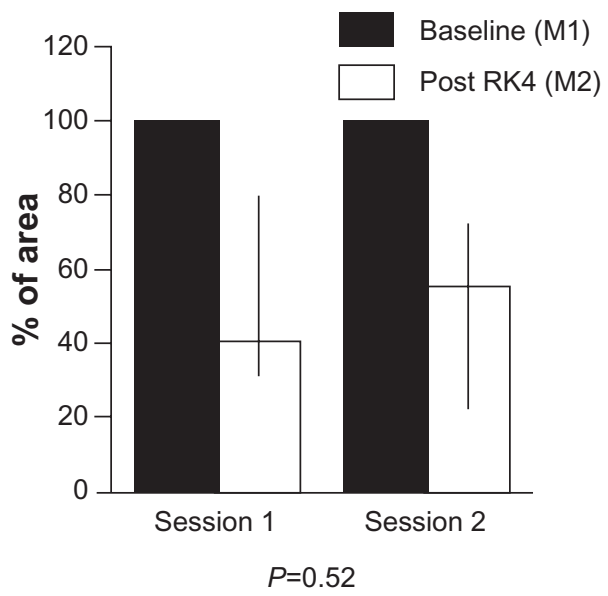

Treatment group (part B - gabapentin or placebo)

Area of secondary hyperalgesia

(to VF stimulation)

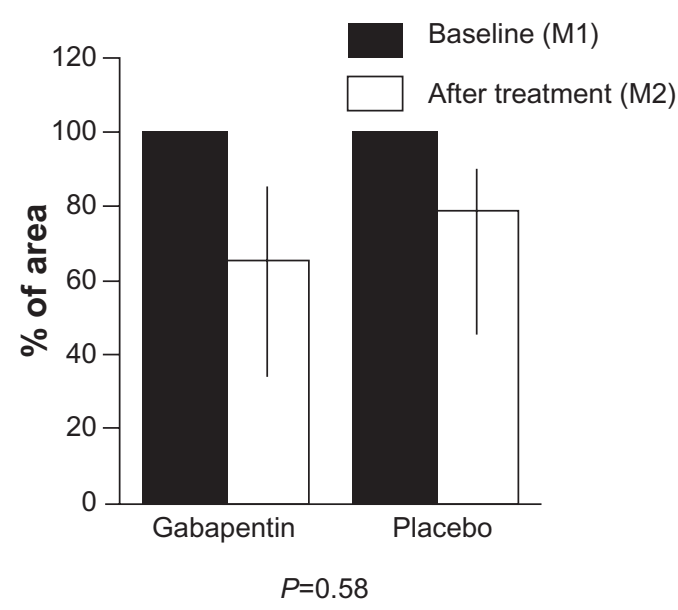

Area of secondary allodynia (to brush stimulation)

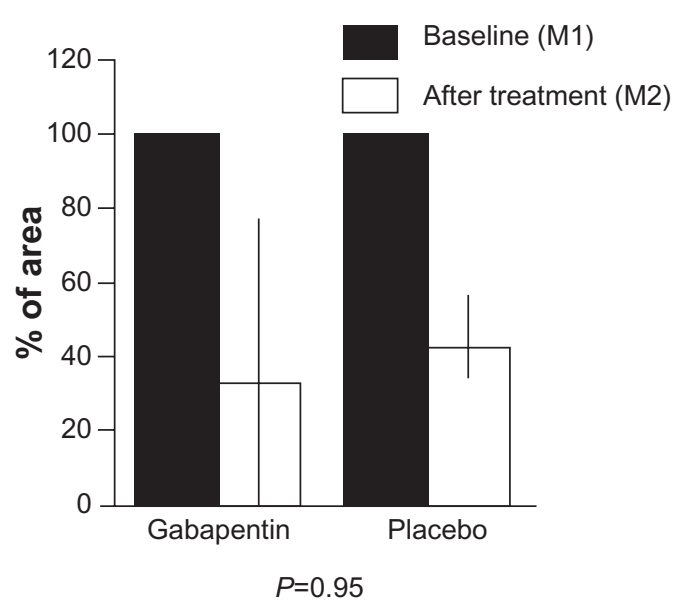

Figure 3 Areas of secondary hyperalgesia and allodynia.

Notes: Percentage change of area size after treatment in the placebo/gabapentin group and at the same time-point, after the fourth rekindling, in the control group (M2) compared to baseline (MI); data are presented as medians, Ist, and 3rd quartiles; $P$-values refer to comparison of percentage changes in VF and brush areas between sessions (control group) and between treatments (placebo/gabapentin group). A $P$-value less than 0.05 was considered statistically significant.

Abbreviations: RK, rekindling; VF, von Frey.

was performed with one-way ANOVA to test for differences of VAS over time; 5 time-points, 1 minute apart, were considered). In the absence of significant differences in VAS scores recorded in the course of the 5 minutes, it was decided to use cumulative mean VAS scores obtained during RK1 and
RK4 to evaluate potential differences between groups (mean VAS score during RK1 and RK4) and within group (mean VAS score at RK1 versus mean VAS score at RK4).

In the control group (Part A), the mean VAS score over 5 minutes at RK1 was 32 (standard deviation [SD] 20.3) in 


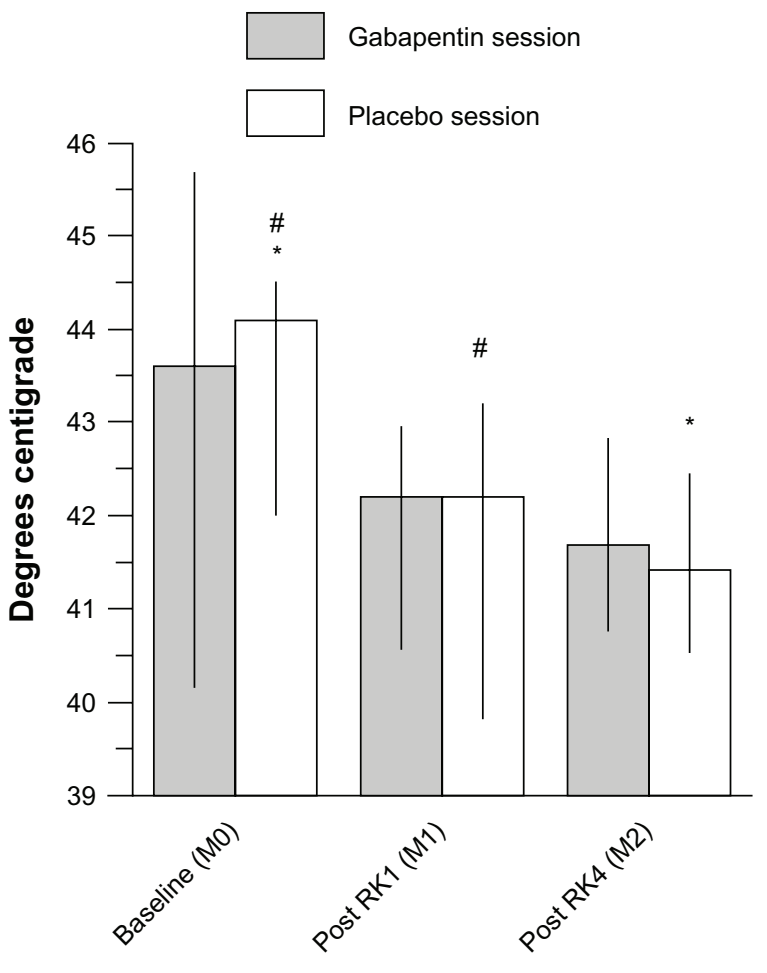

Figure 4 HPDTs in the treatment group.

Notes: HPDT changes at the first and second set of measurements (MI and $M 2$, respectively) are compared to presensitization baseline (M0) in the gabapentin and the placebo sessions; data are presented as medians, Ist, and 3rd quartiles; significantly different couples of measurements are indicated: ${ }^{*} P=0.03 ; * P=0.002$. (A $P$-value less than 0.05 was considered statistically significant). In the placebo group HPDTs remained significantly different than baseline at each time point after induction of heat/capsaicin sensitization.

Abbreviations: HPDT, heat pain detection thresholds; RK, rekindling.

session 1 and 32.5 (SD 22.5) in session 2. The mean VAS score over 5 minutes at RK4 was 15.3 (SD 15.7) in session 1 and 9.9 (SD 12.8) in session 2.

In the treatment group (Part B), the mean VAS score at RK1 was 40.2 (SD 18) on the placebo day and 40.4 (SD 21.5) on the gabapentin day, and the mean VAS score at RK4 was 21.3 (SD 19.8) on the placebo day and 19.7 (SD 18.3) on the gabapentin day.

VAS scores at RK4 were significantly lower than scores at RK1 in all sessions, independent of the treatment (or no treatment). In particular, in Part A, both in session 1 and session 2, VAS scores during RK4 were lower than scores during RK1 (session 1: $P<0.0025$ and session 2: $P<0.0009$; Figure 6A). In Part B, both on placebo and gabapentin day RK4 (post-drug) VAS scores were significantly lower than RK1 scores $(P<0.00005$ and $P<0.00001$, respectively; Figure 6B).

\section{PTS}

\section{Study sessions (part A and part B)}

In both Part $\mathrm{A}$ and Part $\mathrm{B}$, median and maximum pain scores measured by VAS were not significantly different after heat and capsaicin application compared to baseline (M0) at any time point (M1 and M2). In particular, in Part B, as expected, painfulness did not differ after placebo or gabapentin administration $(P=0.13) .{ }^{16}$

\section{Plasma concentrations of gabapentin}

Median plasma concentration measured 150 minutes after drug administration (post-M2 in Figure 1B) was $4.3 \mu \mathrm{g} / \mathrm{mL}$ (3.1-6.0 $\mu \mathrm{g} / \mathrm{mL}$ ). There was no correlation between plasma concentration of gabapentin and reduction of areas of hyperalgesia $\left(R^{2}=0.003\right)$ or allodynia $\left(R^{2}=0.035\right)$.

\section{Discussion}

In our study, areas of sensitization to VF and brush stimulation faded over time independent of the treatment administered (gabapentin or placebo), and also in the absence of any treatment (control group). Interestingly, the spontaneous fading of areas despite rekindling procedures (Figure 5) was consistent with the previously demonstrated spontaneous decay of these areas in the absence of ongoing heat stimulation. ${ }^{10}$ In the original study by Petersen and Rowbotham, ${ }^{10}$ in the absence of rekindling $\left(40^{\circ} \mathrm{C}\right.$ for 5 minutes every 40 minutes for over 3 hours), the areas of secondary hyperalgesia and allodynia "decreased steadily during the study period, demonstrating the necessity of ongoing nociceptor input." Indeed, in our study, the area sizes decreased steadily despite "ongoing nociceptor input" whereas, in the original study, rekindling procedures maintained stable areas of hypersensitivity "for at least 4 hours" after induction of sensitization. ${ }^{10}$

As previously observed, ${ }^{1,10,17,31}$ we also found that brush areas were consistently smaller than VF areas at each time point. In contrast with prior observations, ${ }^{10}$ initial areas of hyperalgesia to VF stimulation obtained in session 2 (as measured post-RK1) were smaller than areas obtained in session $1(P=0.02)$, while the difference in initial brush areas between the two sessions did not reach statistical significance $(P=0.09$; Figure 5). The difference in areas of hyperalgesia between the two sessions may simply reflect the instability of these areas, while the absence of a significant difference in areas of mechanical allodynia between sessions may indicate a lack of power of our study to detect small differences between these already small areas. As previously reported, intrinsic differences in the type of applied mechanical stimuli underlie the different behavior of the areas obtained: brush stimulation is thought to activate mainly $A \beta$ fibers, whereas VF filaments stimulation probably activates mainly A $\delta$ fibers. ${ }^{10}$ 
A

\section{Brush areas control group session 1}

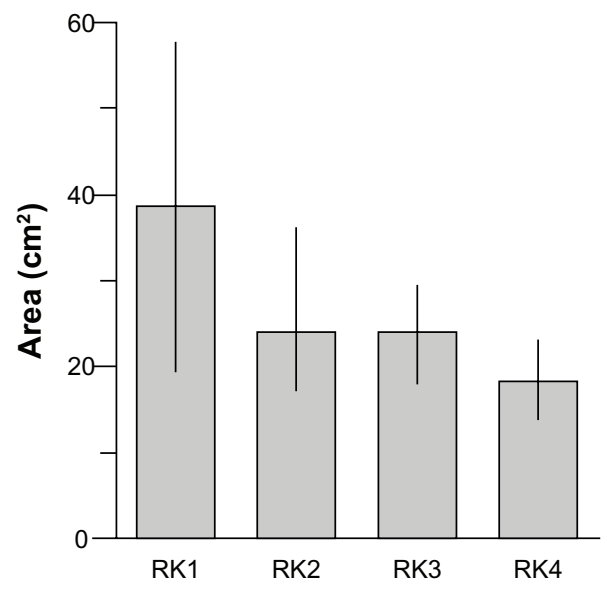

C von Frey areas
control group session 1

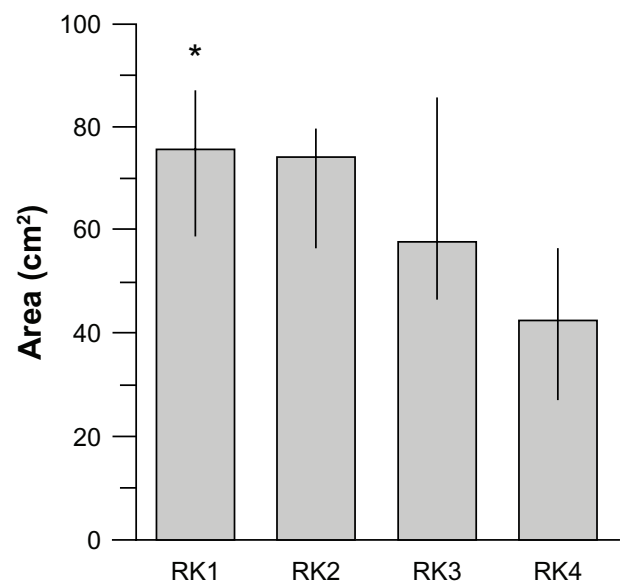

B

\section{Brush areas control group session 2}

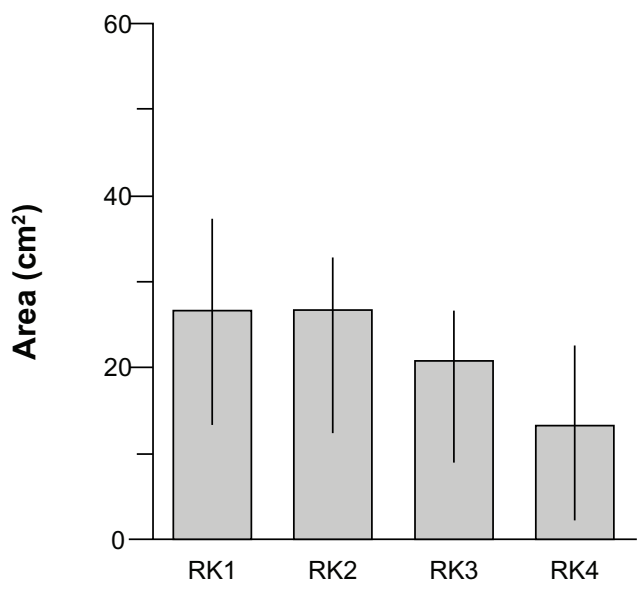

D

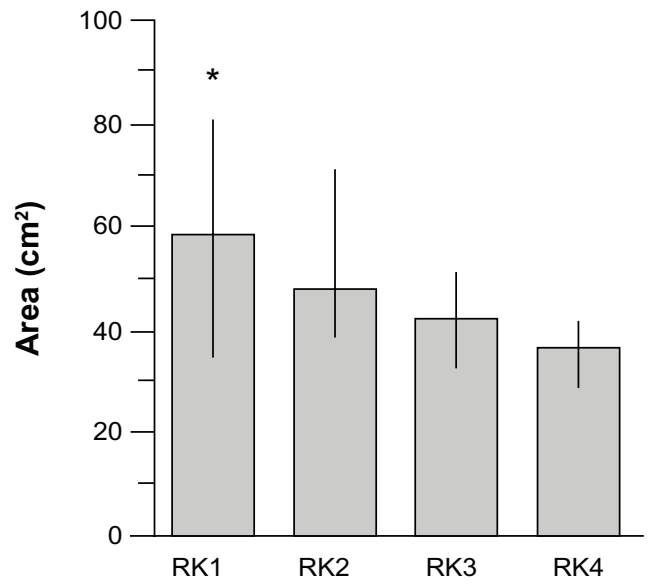

Figure 5 Brush and von Frey areas: spontaneous fading despite rekindling procedure in control group.

Notes: Spontaneous fading of Brush areas (A and $\mathbf{B})$ and von Frey areas (C and $\mathbf{D})$ despite rekindling procedures is shown in the control group. Data are presented as medians, Ist, and 3 rd quartiles. Initial von Frey areas in session 2 are significantly smaller than session I: $* P<0.02$ (A P-value less than 0.05 was considered statistically significant).

Abbreviation: RK, rekindling.

Significantly decreased HPDTs after heat and capsaicin application confirmed the creation of primary sensitization with this model, as previously demonstrated. ${ }^{10,16}$ However, unlike previous reports areas of secondary hypersensitivity were not stable. Stable areas would have been necessary to test gabapentin's effectiveness in suppressing established cutaneous sensitization compared to placebo. The persistent reduction of HPDTs after RK4 (M2) compared to baseline presensitization (M0) in the placebo session may indicate a tendency toward reaching an adequate intensity of stimulation to sustain the area of primary heat hyperalgesia through the rekindling procedures. However, a similar effect was not present in the control group.

While the initial heat/capsaicin stimulation was sufficient to create an area of primary hyperalgesia, characterized by hypersensitivity to heat/pain stimulation,,$^{10,31,32}$ the rekindling procedures were not adequate to maintain it.

Previous studies have demonstrated that the size of the primary area of sensitization may be a critical factor in creating and maintaining an area of secondary sensitization to both heat and mechanical stimulation. ${ }^{20,33}$ Three main factors seem to contribute to the development and persistence 
A

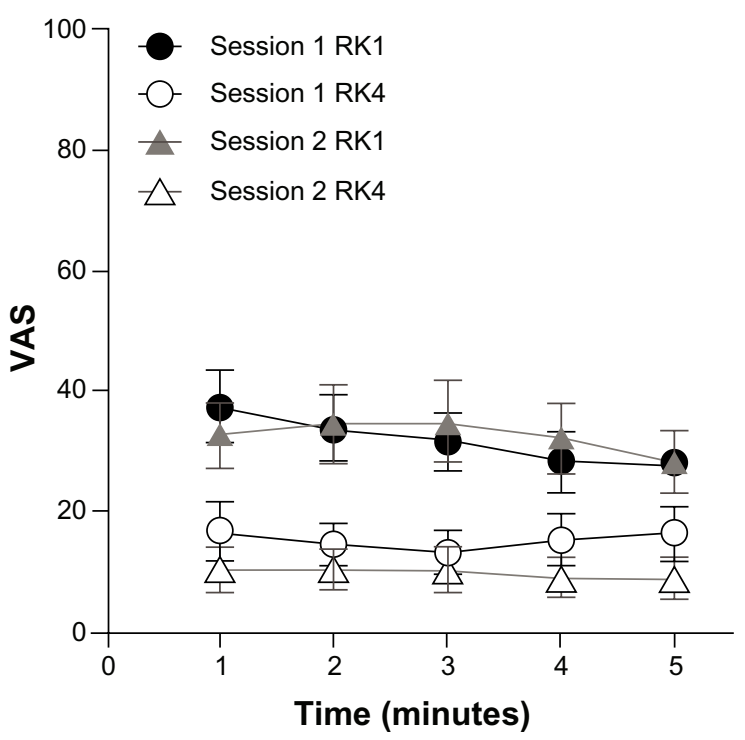

B

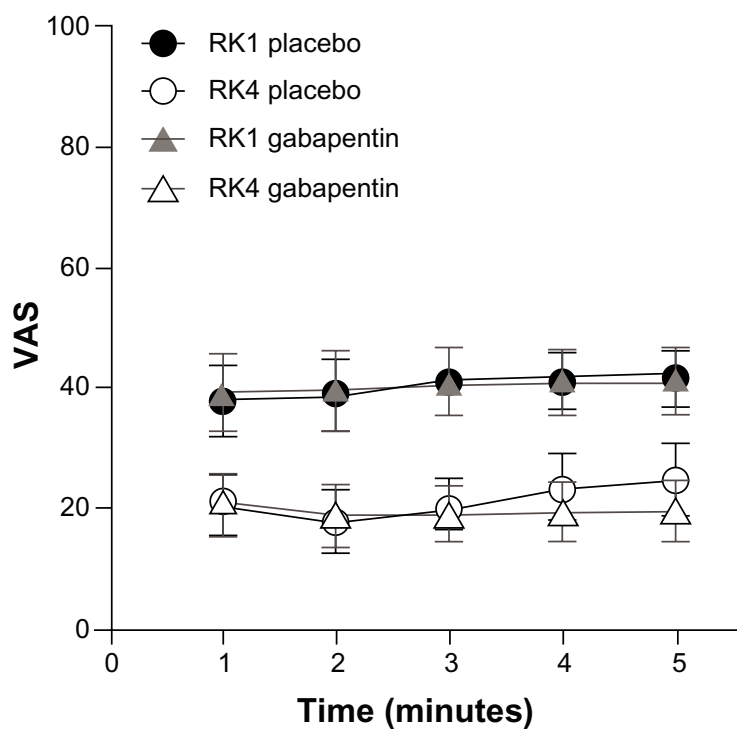

Figure 6 Mean VAS scores for each minute during the 5-minute RK preceding the sets of measurements MI (RKI) and M2 (RK4).

Notes: Mean VAS scores for each minute during the 5-minute RK preceding the sets of measurements MI (RKI) and M2 (RK4). Data are presented as means and standard deviations. (A) Session I and 2 of the control group are compared; (B) Placebo day and gabapentin day of the treatment group are compared. In both groups and both study sessions, painfulness at each minute of the rekindling procedures decreased significantly between the first and fourth rekindling, despite treatment (or no treatment). Part A: both in session I and session 2, VAS scores during RK4 were lower than scores during RKI (session I: $P<0.0025$ and session $2 P<0.0009$ ). Part B: both on placebo and gabapentin day RK4 (post-drug), VAS scores were significantly lower than RKI scores $(P<0.00005$ and $P<0.0000$ I, respectively). A $P$-value less than 0.05 was considered statistically significant.

Abbreviations: RK, rekindling procedure; VAS, visual analog scale.

of areas of secondary sensitization: intensity of the painful stimulation; ${ }^{25}$ duration of the noxious stimulation (continuous or repeated exposure over time); ;,34,35 and size of the area subject to primary sensitization..$^{20,33} \mathrm{An}$ adequate combination of these three elements appears to be critical to maintain a nociceptive input barrage strong enough to sustain central sensitization. Establishing a "critical size" (in absolute or even relative terms) to obtain a "sensitization outcome" of a predictable duration and magnitude with this model - similar to the Minimal Erythema Dose utilized by Gustorff et al in the UVB burn model ${ }^{9,36}$ - may prove difficult due to the contribution of multiple factors to this effect (including time of exposure, intensity of the two noxious stimuli, and intrinsic characteristics of the subject). ${ }^{9,37,38}$ However, much like it has been hypothesized for secondary hyperalgesia to heat, ${ }^{33}$ the development and persistence of secondary hyperalgesia to mechanical stimulation in this experimental model seems to critically depend on the magnitude and intensity of the stimulation in the area of peripheral sensitization. ${ }^{39}$

Yucel et al could not confirm stable areas of secondary hyperalgesia and allodynia by associating heat pre- and postconditioning to topical capsaicin application. ${ }^{20}$ In Yucel et al's study, conditioning with heat in an attempt to "rekindle the spontaneous afferent C-fiber input" did not have any effect on the ongoing pain ratings and sensory test results
(VF and brush stimulation).$^{20}$ Use of a smaller probe, shorter rekindling, and lower temperatures than utilized by Petersen and Rowbotham ${ }^{10}$ were examined by the authors as possible causes of failure to obtain stable areas of hypersensitization. They observed that "the duration and stability of secondary hyperalgesia seems to depend on both the intensity of the initial stimulus and on continued nociceptive input."${ }^{20}$ Our findings are consistent with this observation.

In the absence of sufficient ongoing nociceptive input, areas of secondary hyperalgesia rapidly and spontaneously decline, ${ }^{32}$ making it difficult to appreciate potential differences between treatments aimed at reducing these areas as the differences, proportionally, tend to become smaller over time. Indeed, in our study, areas of secondary hyperalgesia at baseline were smaller than areas previously obtained with this model using larger thermal probes for induction. Our average VF areas tested immediately after induction of heat/capsaicin sensitization (M-test in Figure 1A) were $85 \mathrm{~cm}^{2}$ for female subjects (groups pooled, $\mathrm{n}=16$ ) and $78 \mathrm{~cm}^{2}$ for male subjects $(\mathrm{n}=14)$; brush areas were $43 \mathrm{~cm}^{2}$ for females and $48 \mathrm{~cm}^{2}$ for males. These areas were significantly smaller than the areas obtained from a series of studies conducted with a larger heated probe $\left(15.7 \mathrm{~cm}^{2}\right.$ surface area thermode [Medoc TSA 2001; Medoc Ltd.]). ${ }^{40}$ Data from these studies were pooled to explore gender differences and analyzed by Jensen and 
Petersen. In 85 healthy subjects (41 females/44 males) the mean VF areas were $126 \mathrm{~cm}^{2}$ for female and $153 \mathrm{~cm}^{2}$ for males, and the mean brush area was $107 \mathrm{~cm}^{2}$ for females and $112 \mathrm{~cm}^{2}$ for males. ${ }^{40}$

The area of secondary hyperalgesia at baseline has been considered a determinant of assay sensitivity in the intradermal capsaicin model. ${ }^{22}$ Using this model to evaluate the effect of different treatments on capsaicin induced secondary hyperalgesia and allodynia, Wang et al found that increasing precision for discrimination of active treatment from placebo was associated with increasing the area of secondary hyperalgesia at screening of the subjects. ${ }^{22}$

Our selection criteria for healthy volunteers, study procedures, and all other measurable variables, other than the smaller size thermode, were consistent with those used in prior placebo-controlled studies in which the model created stable, long-lasting areas of hypersensitivity and secondary hyperalgesia, adequate to test the effect of different drugs on experimental cutaneous hyperalgesia., ${ }^{2,15,16}$ Therefore, our hypothesis is that the main contributing factor to our inability to maintain stable areas of sensitization was the relatively undersized thermode, which produced smaller areas of hypersensitivity, and failed to produce adequate nociceptive input to maintain these areas through the rekindling procedures.

When this smaller thermode is used to produce cutaneous sensitization in association with capsaicin, a sufficient ongoing nociceptive input might be provided by prolonging exposure to capsaicin ${ }^{28}$ or increasing the number and frequency of the rekindling procedures. In a functional magnetic resonance imaging (fMRI) study on pharmacological modulation of pain-related brain activity during normal and central sensitization states, Iannetti et al ${ }^{28}$ utilized the $9 \mathrm{~cm}^{2}$ thermode to produce heat/capsaicin cutaneous sensitization on the shin area of the legs of 12 healthy male volunteers. The main finding of this study is that gabapentin has a major modulatory effect on fMRI brain responses to nociceptive inputs during central sensitization. ${ }^{28}$

In contrast to our study, Iannetti et $\mathrm{al}^{28}$ administered gabapentin before induction of sensitization, and heat and capsaicin were applied at the time of the expected plasmatic peak of gabapentin. In addition, the fMRI scan was started only 1 hour after induction of the model, and the capsaicin cream was not removed from the skin until the end of the scanning period, resulting in a total time of capsaicin application of approximately 100 minutes versus 30 minutes in the study reported here. In these conditions, which were modified from the original model, the cutaneous sensitization was maintained by extending the exposure to capsaicin.
The effects of gabapentin in modulating brain activity in enhanced pain states were evaluated by fMRI activity during this "prolonged induction" phase, and no rekindling procedures were performed to maintain the sensitization after this initial phase.

Baseline pain measured with VAS during PTS on the nondominant upper arm (LTS [long thermal stimulation] in the original study by Dirks et al) ${ }^{16}$ was not different between groups on the training day nor on any of the study days. Pain scoring was not significantly different after heat and capsaicin application compared to baseline (pre-heat/capsaicin application) in any group, and, as expected, ${ }^{16,41}$ painfulness did not differ after placebo or gabapentin administration. Similarly, in the area of primary sensitization, no difference was noted in pain scores obtained during matched rekindling procedures between sessions (eg RK1 versus RK1 in different study days), and, regardless of the treatment, VAS scores during RK4 were lower than VAS scores during RK1.

Arguably, our plasma concentrations of gabapentin (average $4.3 \mu \mathrm{g} / \mathrm{mL}$ ) might have been too low to detect a significant effect of gabapentin compared to placebo in our conditions. ${ }^{41,42}$ However, our concentrations were in the range of therapeutic plasma concentrations $(2-15 \mu \mathrm{g} / \mathrm{mL})$, and the route, dose, and administration schedule that we used were consistent with the original protocol that we sought to reproduce, ${ }^{16}$ where gabapentin demonstrated significant antihyperalgesic efficacy.

Since we did not have a comparably sized thermode as described in prior studies, we were not able to test whether we could reproduce the model with a larger thermal probe. Indeed, the fact that larger heating probes are not currently commercially available was one of the reasons why we were interested in assessing whether a smaller probe could reproduce reported results obtained with this model.

\section{Conclusion}

The ability to create long-lasting and stable areas of sensitization set the heat/capsaicin model apart from other models of experimental cutaneous sensitization, and made it suitable to study reversal of established hyperalgesia. The heat/capsaicin model, as originally proposed, has become widely popular, and extensively reported and utilized in pain research. However, size, duration, and intensity of noxious stimulation have been shown to be critical for the induction and maintenance of areas of secondary hyperalgesia in multiple conditions. In our experimental setting, all other controllable variables were consistent with the original protocol that we were seeking to replicate, except for the use of 
a smaller size thermode, and, in these conditions, we could not confirm previous findings of a significant difference between gabapentin and placebo in reducing areas of heat/ capsaicin induced cutaneous sensitization. We hypothesized that the reduction in area of sensitization observed after placebo might depend on a spontaneous fading of the area as opposed to a "placebo effect", which had not been previously observed.

To our knowledge, this is the first study demonstrating the inability to reproduce the original heat/capsaicin cutaneous sensitization model by applying the critical "ongoing nociceptive input" on a smaller surface area than originally reported. Additionally, we hope that our negative result will bring focus on the significant issue of standardizing and validating experimental pain models. The advancement of translational pain research strictly depends on the availability of experimental pain models and methods that can be easily and consistently reproduced, so that they can be uniformly applied and provide results that are significant, predictive of clinical efficacy, and comparable among different studies.

\section{Acknowledgments}

Funding for this study was provided by a Pilot Award from the Barnes Jewish Hospital Foundation/ICTS Clinical and Translational Funding Program (supported by NIH CTSA Grant \# UL1 RR024992), and by grant NIH R01NS048602 as well as R01NS048602-11S1 from the National Institute of Neurological Disorders and Stroke to Robert W Gereau IV.

\section{Disclosure}

The authors report no conflicts of interest in this work.

\section{References}

1. Dirks J, Petersen KL, Dahl JB. The heat/capsaicin sensitization model: a methodologic study. J Pain. 2003;4(3):122-128.

2. Frymoyer AR, Rowbotham MC, Petersen KL. Placebo-controlled comparison of a morphine/dextromethorphan combination with morphine on experimental pain and hyperalgesia in healthy volunteers. J Pain. 2007;8(1):19-25.

3. Staud R. Evidence for shared pain mechanisms in osteoarthritis, low back pain, and fibromyalgia. Curr Rheumatol Rep. 2011;13(6):513-520.

4. Steyaert A, De Kock M. Chronic postsurgical pain. Curr Opin Anaesthesiol. 2012;25(5):584-588.

5. Staud R. Is it all central sensitization? Role of peripheral tissue nociception in chronic musculoskeletal pain. Curr Rheumatol Rep. 2010;12(6):448-454.

6. Staud R. Brain imaging in fibromyalgia syndrome. Clin Exp Rheumatol. 2011;29(6 Supp1 69):S109-S117.

7. Staud R. Peripheral pain mechanisms in chronic widespread pain. Best Pract Res Clin Rheumatol. 2011;25(2):155-164.

8. Graeber MB, Christie MJ. Multiple mechanisms of microglia: a gatekeeper's contribution to pain states. Exp Neurol. 2012;234(2): $255-261$.
9. Gustorff B, Anzenhofer S, Sycha T, Lehr S, Kress HG. The sunburn pain model: the stability of primary and secondary hyperalgesia over 10 hours in a crossover setting. Anesth Analg. 2004;98(1):173-177, table of contents.

10. Petersen KL, Rowbotham MC. A new human experimental pain model: the heat/capsaicin sensitization model. Neuroreport. 1999;10(7): 1511-1516.

11. Dirks J, Petersen KL, Rowbotham MC, Dahl JB. Effect of systemic adenosine on pain and secondary hyperalgesia associated with the heat/ capsaicin sensitization model in healthy volunteers. Reg Anesth Pain Med. 2001;26(5):414-419.

12. Dirks J, Fabricius P, Petersen KL, Rowbotham MC, Dahl JB. The effect of systemic lidocaine on pain and secondary hyperalgesia associated with the heat/capsaicin sensitization model in healthy volunteers. Anesth Analg. 2000;91(4):967-972.

13. Petersen KL, Maloney A, Hoke F, Dahl JB, Rowbotham MC. A randomized study of the effect of oral lamotrigine and hydromorphone on pain and hyperalgesia following heat/capsaicin sensitization. J Pain. Sep 2003;4(7):400-406.

14. Abrams DI, Jay CA, Shade SB, et al. Cannabis in painful HIV-associated sensory neuropathy: a randomized placebo-controlled trial. Neurology. 2007;68(7):515-521.

15. Petersen KL, Jones B, Segredo V, Dahl JB, Rowbotham MC. Effect of remifentanil on pain and secondary hyperalgesia associated with the heat - capsaicin sensitization model in healthy volunteers. Anesthesiology. 2001;94(1):15-20.

16. Dirks J, Petersen KL, Rowbotham MC, Dahl JB. Gabapentin suppresses cutaneous hyperalgesia following heat-capsaicin sensitization. Anesthesiology. 2002;97(1):102-107.

17. Hughes A, Macleod A, Growcott J, Thomas I. Assessment of the reproducibility of intradermal administration of capsaicin as a model for inducing human pain. Pain. 2002;99(1-2):323-331.

18. Staahl C, Olesen AE, Andresen T, Arendt-Nielsen L, Drewes AM. Assessing efficacy of non-opioid analgesics in experimental pain models in healthy volunteers: an updated review. Br J Clin Pharmacol. 2009;68(3):322-341.

19. Oertel BG, Lotsch J. Clinical pharmacology of analgesics assessed with human experimental pain models: bridging basic and clinical research. Br J Pharmacol. 2013;168(3):534-553.

20. Yucel A, Miyazawa A, Andersen OK, Arendt-Nielsen L. The effect of heat conditioning of the primary area before and after induction of hyperalgesia by topical/intradermal capsaicin or by controlled heat injury. Somatosens Mot Res. 2001;18(4):295-302.

21. Pedersen JL, Kehlet H. Hyperalgesia in a human model of acute inflammatory pain: a methodological study. Pain. 1998;74(2-3): 139-151.

22. Wang H, Bolognese J, Calder N, et al. Effect of morphine and pregabalin compared with diphenhydramine hydrochloride and placebo on hyperalgesia and allodynia induced by intradermal capsaicin in healthy male subjects. J Pain. 2008;9(12):1088-1095.

23. Modir JG, Wallace MS. Human Experimental Pain Models 3: Heat/CapsaicinSensitization and Intradermal Capsaicin Models. In: Szallasi A, editor. Analgesia: Methods and Protocols, Methods in Molecular Biology. New York: Humana Press; 2010;617:169-174.

24. Petersen K, Schmelz M. Human Pain Models: Virtues and Limitations. Paper presented at: IASP Refresher Course presented at the 12th World Congress on Pain August 17-22, 2008; Glasgow, Scotland, UK.

25. LaMotte RH, Lundberg LE, Torebjörk HE. Pain, hyperalgesia and activity in nociceptive $\mathrm{C}$ units in humans after intradermal injection of capsaicin. J Physiol. 1992;448:749-764.

26. Koltzenburg M, Torebjörk HE, Wahren LK. Nociceptor modulated central sensitization causes mechanical hyperalgesia in acute chemogenic and chronic neuropathic pain. Brain. 1994;117(Pt 3): 579-591.

27. Hood DD, Curry R, Eisenach JC. Intravenous remifentanil produces withdrawal hyperalgesia in volunteers with capsaicin-induced hyperalgesia. Anesth Analg. 2003;97(3):810-815. 
28. Iannetti GD, Zambreanu L, Wise RG, et al. Pharmacological modulation of pain-related brain activity during normal and central sensitization states in humans. Proc Natl Acad Sci U S A. 2005;102(50):18195-18200.

29. Campbell CM, Bounds SC, Simango MB, et al. Self-reported sleep duration associated with distraction analgesia, hyperemia, and secondary hyperalgesia in the heat-capsaicin nociceptive model. Eur J Pain. 2011;15(6):561-567.

30. Eisenach JC, Curry R, Tong C, Houle TT, Yaksh TL. Effects of intrathecal ketorolac on human experimental pain. Anesthesiology. 2010;112(5):1216-1224.

31. LaMotte RH, Shain CN, Simone DA, Tsai EF. Neurogenic hyperalgesia: psychophysical studies of underlying mechanisms. J Neurophysiol. 1991;66(1):190-211.

32. Moiniche S, Dahl JB, Kehlet H. Time course of primary and secondary hyperalgesia after heat injury to the skin. Br J Anaesth. 1993;71(2): 201-205.

33. Pedersen JL, Kehlet H. Secondary hyperalgesia to heat stimuli after burn injury in man. Pain. 1998;76(3):377-384.

34. Cervero F, Gilbert R, Hammond RG, Tanner J. Development of secondary hyperalgesia following non-painful thermal stimulation of the skin: a psychophysical study in man. Pain. 1993;54(2):181-189.

35. Andersen OK, Jensen LM, Brennum J, Arendt-Nielsen L. Evidence for central summation of $\mathrm{C}$ and A delta nociceptive activity in man. Pain. 1994;59(2):273-280.
36. Gustorff B, Hauer D, Thaler J, Seis A, Draxler J. Antihyperalgesic efficacy of $5 \%$ lidocaine medicated plaster in capsaicin and sunburn pain models - two randomized, double-blinded, placebo-controlled crossover trials in healthy volunteers. Expert Opin Pharmacother. 2011;12(18):2781-2790.

37. Klein T, Magerl W, Hanschmann A, Althaus M, Treede RD. Antihyperalgesic and analgesic properties of the N-methyl-D-aspartate (NMDA) receptor antagonist neramexane in a human surrogate model of neurogenic hyperalgesia. Eur J Pain. 2008;12(1):17-29.

38. O'Neill J, Brock C, Olesen AE, Andresen T, Nilsson M, Dickenson AH. Unravelling the mystery of capsaicin: a tool to understand and treat pain. Pharmacol Rev. 2012;64(4):939-971.

39. Torebjörk HE, Lundberg LE, LaMotte RH. Central changes in processing of mechanoreceptive input in capsaicin-induced secondary hyperalgesia in humans. J Physiol. 1992;448:765-780.

40. Jensen MT, Petersen KL. Gender differences in pain and secondary hyperalgesia after heat/capsaicin sensitization in healthy volunteers. J Pain. 2006;7(3):211-217.

41. Wallace MS, Schulteis G. Effect of chronic oral gabapentin on capsaicin-induced pain and hyperalgesia: a double-blind, placebocontrolled, crossover study. Clin J Pain. 2008;24(6):544-549.

42. Gottrup H, Juhl G, Kristensen AD, et al. Chronic oral gabapentin reduces elements of central sensitization in human experimental hyperalgesia. Anesthesiology. 2004;101(6):1400-1408.
Journal of Pain Research

\section{Publish your work in this journal}

The Journal of Pain Research is an international, peer-reviewed, open access, online journal that welcomes laboratory and clinical findings in the fields of pain research and the prevention and management of pain. Original research, reviews, symposium reports, hypothesis formation and commentaries are all considered for publication.

\section{Dovepress}

The manuscript management system is completely online and includes a very quick and fair peer-review system, which is all easy to use. Visit http://www.dovepress.com/testimonials.php to read real quotes from published authors. 